\title{
A family of phase masks for broadband coronagraphy example of the wrapped vortex phase mask theory and laboratory demonstration
}

\author{
R. Galicher, E. Huby, P. Baudoz, and O. Dupuis
}

\begin{abstract}
LESIA, Observatoire de Paris, PSL Research University, CNRS, Sorbonne Universités, Univ. Paris Diderot, UPMC Univ. Paris 06, Sorbonne Paris Cité, 5 place Jules Janssen, 92190 Meudon, France

e-mail: raphael.galicher@obspm.fr
\end{abstract}

Received 22 October 2019 / Accepted 13 January 2020

\begin{abstract}
Context. Future instruments need efficient coronagraphs over large spectral ranges to enable broadband imaging or spectral characterization of exoplanets that are $10^{8}$ times fainter than their star. Several solutions have been proposed. Pupil apodizers can attenuate the star intensity by a factor of $10^{10}$ but they only transmit a few percent of the light of the planet. Cascades of phase and/or amplitude masks can both attenuate the starlight and transmit most of the planet light, but the number of optics that require alignment makes this solution impractical for an instrument. Finally, vector phase masks can be used to detect faint sources close to bright stars but they require the use of high-quality circular polarizers and, as in the previous solution, this leads to a complex instrument with numerous optics that require alignment and stabilization.

Aims. We propose simple coronagraphs that only need one scalar phase mask and one binary Lyot stop providing high transmission for the planet light $(>50 \%)$ and high attenuation of the starlight over a large spectral bandpass $(\sim 30 \%)$ and a $360^{\circ}$ field-of-view.

Methods. From mathematical considerations, we find a family of 2D phase masks optimized for an unobscured pupil. One mask is an azimuthal wrapped vortex phase ramp. We probe its coronagraphic performance using numerical simulations and laboratory tests. Results. From numerical simulations, we predict the wrapped vortex can attenuate the peak of the star image by a factor of $10^{4}$ over a $29 \%$ bandpass and $10^{5}$ over a $18 \%$ bandpass with transmission of more than $50 \%$ of the planet flux at $\sim 4 \lambda / D$. We confirm these predictions in the laboratory in visible light between 550 and $870 \mathrm{~nm}$. We also obtain laboratory dark hole images in which exoplanets with fluxes that are $3 \times 10^{-8}$ times the host star flux could be detected at $3 \sigma$.

Conclusions. Taking advantage of a new technology for etching continuous 2D functions, a new type of mask can be easily manufactured opening up new possibilities for broadband coronagraphy.
\end{abstract}

Key words. instrumentation: high angular resolution - techniques: high angular resolution - planets and satellites: detection

\section{Introduction}

Exoplanets and disks are objects that we study to understand the formation of planetary systems. To date, more than 4000 exoplanets have been detected. Statistics of their orbital parameters (Santos 2008; Santos \& Faria 2018) put constraints on the models that explain the formation of planets (Raymond et al. 2014; Izidoro \& Raymond 2018; Santos et al. 2017; D'Angelo \& Lissauer 2018; Adibekyan 2019). To put more constraints on these models, we require detections of exoplanets that orbit in the outer part of their system, close to the ice-line. However, the most commonly used detection techniques - radial velocity and transit - do not easily observe such exoplanets. Another challenge is the study of the exoplanet atmospheres and surface temperatures, both of which require the measurement of the planet spectrum in transmission, reflection, or emission. The transit technique enables detections of molecules in the atmosphere of exoplanets in favorable cases (Snellen et al. 2010; Alonso-Floriano et al. 2019; von Essen et al.2019; Espinoza et al. 2019), but transits focus on exoplanets orbiting very close to their star. For nontransiting planets like $\beta$-Pictoris b or HR $8799 \mathrm{e}$, medium- to high-resolution spectra have been used to detect molecules (Snellen et al. 2014; Gravity Collaboration 2019) but such techniques can only be used to study known exoplanets. The discovery of planets in the outer part of their system therefore requires direct imaging techniques working on broad spectral bands. Statistical analyses of these detections put constraints on the models of planet formation. Imaging also allows spectral characterization that can be used to study exoplanet atmospheres.

Direct imaging is very challenging because exoplanets are up to $10^{10}$ times fainter than their host star in visible and infrared. It requires dedicated instrumentation composed of extreme adaptive optics systems and coronagraphs, as in the current instruments Spectro-Polarimetric High-contrast Exoplanet REsearch instrument at the Very Large Telescope (Beuzit et al. 2019), Gemini Planet Imager (Macintosh et al. 2014), SCExAO/Subaru (Lozi et al. 2018), and Magellan adaptive optics instrument (Close et al. 2018). These instruments have enabled the detection of a few young giant exoplanets and numerous circumstellar disks of dust. However, none of them can attenuate the star intensity by more than a factor of $10^{3}$ over a large spectral range for two main reasons. Optical aberrations induce speckles in the science image. Some of them are calibrated a posteriori using differential imaging (Racine et al. 1999; Baba \& Murakami 2003; Marois et al. 2006) and in the near future their intensity will 


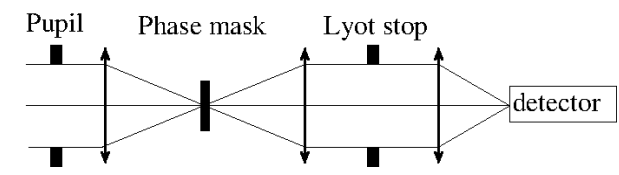

Fig. 1. Principle of a coronagraph. The on-axis source image is centered on a focal plane phase mask that scatters the light outside the geometrical pupil in the following plane. The Lyot stop filters out the starlight that does not reach the detector.

be actively minimized thanks to focal plane wavefront sensing (Jovanovic et al. 2018). The second limitation comes from the current coronagraphs themselves because there is a trade-off between starlight attenuation and the spectral bandpass of the coronagraph.

For future instruments that aim to characterize exoplanets such as Earth or mature Jupiter, new broadband coronagraphs are required with high attenuation of the starlight. Several solutions have been proposed and a few of them were validated in the laboratory, attenuating the starlight by a factor of $10^{8}-10^{9}$ (Galicher et al. 2011; Mawet et al. 2011; Serabyn et al. 2019). No solution is perfect and trade-offs remain. For example, for the shaped pupil coronagraphs, a comprimise must be made between the field of view, the attenuation of the starlight, and the transmission of the exoplanet flux (Cady et al. 2017). In this paper, we present a family of coronagraphs based on only one scalar phase mask. These are efficient over a 30\% spectral band with a very high transmission of the planet intensity in a $360^{\circ}$ field of view. In Sect. 2, we briefly review the current limitations of phase mask coronagraphy and we use a mathematical approach to derive the phase functions of a family of scalar broadband coronagraphic masks. One of these masks is the wrapped vortex phase mask that we study using numerical simulations in Sect. 3. We then present our wrapped vortex prototype in Sect. 4 and its laboratory performance in Sect. 5 .

\section{Theory of wrapped broadband phase masks}

\subsection{Phase mask for coronagraphy}

We consider a coronagraph composed of a single focal plane phase mask followed by a classical binary Lyot stop (Fig. 1). The star image is centered on the mask. We call $r$ and $\theta$ the polar coordinates in this plane. If the phase function $G(r, \theta)$ is well chosen, all the starlight is rejected outside of the geometrical pupil in the following pupil plane and it is stopped by the binary Lyot stop. The starlight is filtered out from the system and does not reach the detector. The light of an off-axis source is not focused on the center of the phase mask; it goes through the system, reaches the detector, and its image is detected. Designing such a coronagraph requires finding the $2 \mathrm{D}$ phase function $G(r, \theta)$ that maximizes both the starlight attenuation and the transmission of an off-axis source. Numerous phase functions that theoretically stop $100 \%$ of the starlight have been proposed (Rouan et al. 2000; Mawet et al. 2005; Murakami et al. 2008 etc.).

However, it is difficult to fabricate a component that adds the perfect phase $G_{0}$ at all wavelengths regardless of the type of mask: transmissive or reflective scalar masks or vector phase mask. A transmissive scalar phase mask is made of a material whose thickness $e(r, \theta)$ varies in the field of view. The variations in thickness induce variations in the phase shift $G(r, \theta)=$ $2 \pi(n(\lambda)-1) e(r, \theta) / \lambda$ with $n(\lambda)$ being the optical index of the material at wavelength $\lambda$. The thickness $e$ can therefore be chosen so that the phase $G$ is equal to the "perfect" $G_{0}$ at one wavelength $\lambda_{0}$ which we henceforth refer to as the optimized wavelength. At all other wavelengths, the phase function $G$ differs from the perfect function $G_{0}$ and the starlight is not totally filtered out by the coronagraph. Such a phase mask is therefore monochromatic. A reflective phase mask is also based on variations of the thickness $e(r, \theta)$ inducing variations of the phase $G(r, \theta)=4 \pi e(r, \theta) / \lambda$. As for the transmissive mask, the function $G$ is equal to the perfect function $G_{0}$ at one wavelength $\lambda_{0}$ only.

To mitigate the chromatic dependence of scalar masks, Galicher et al. (2011) and Mawet et al. (2011) proposed the use of several monochromatic phase masks in cascade. This concept enables the starlight to be attenuated over $\sim 20 \%$ of the spectral bandpass but both of these latter two groups of authors concluded that aligning two or three coronagraphs in cascade is too demanding in terms of instrument stability.

Other phase masks are vectorial masks (Mawet et al. 2009), which use a half-wave plate whose neutral axes rotate in the field of view so that the mask induces a geometrical phase shift $G(r, \theta)$. However, the phase shift is polarization dependent and is not perfect because of retardance errors (chromaticity and errors on the orientation of neutral axes). Therefore, the phase function $G$ differs from the perfect function $G_{0}$ and part of the starlight leaks through the coronagraph. It has been shown that the leakage can be minimized if the vector phase mask is set between circular polarizers (Serabyn et al. 2019). However, this removes half of the putative exoplanet flux (removing one of the two polarization states) and requires the use and alignment of polarizers, which is not the optimal solution in terms of instrument stability.

\subsection{General formalism for scalar azimuthal phase masks}

To reduce the complexity of the instrument and maintain the high performance of the coronagraph, we consider a scalar phase mask. Furthermore, to mitigate the wavelength dependance of the phase shift induced by a scalar mask, we look for a $G(r, \theta, \lambda)$ function that is optimized to cancel the starlight over a broadband. In the rest of the paper, we assume that the optical index $n$ of the mask material does not vary with wavelength. For example, the optical index of fused silica is constant at $0.3 \%$ between 600 and $800 \mathrm{~nm}$. We also assume an azimuthal phase mask, meaning the phase function $G$ does not vary with the radial coordinate $r$ :

$G(\theta, \lambda)=\frac{2 \pi \alpha e(r, \theta)}{\lambda}=G_{0}(\theta) \frac{\lambda_{0}}{\lambda}$

The parameter $\alpha$ is a constant that is equal to $n-1$ for a transmissive mask and 2 for a reflective mask. The function $G_{0}$ is the "perfect" phase function that is induced at the optimized wavelength $\lambda_{0}$.

Considering a full entrance pupil (no spider or obscuration), Ma et al. (2012) expressed the amplitude of the stellar electric field in the Lyot plane as a sum of Hankel transforms of Bessel functions. These latter authors also demonstrated that if the $G_{0}$ function is $\pi$-periodic, the sum reduces to a single term proportional to:

$c_{0}(\lambda)=\frac{1}{2 \pi} \int_{0}^{\pi} \exp \left(i G_{0}(\theta) \lambda_{0} / \lambda\right) \mathrm{d} \theta$.

In the rest of the paper, we focus on $\pi$-periodic $G_{0}$ functions that minimize $c_{0}(\lambda)$ - i.e. the starlight leakage - over a large spectral bandpass centered on the optimized wavelength $\lambda_{0}$. To ensure a weak chromatic dependence of the coronagraphic 
performance, Ma et al. (2012) looked for $G_{0}$ functions that cancel out $c_{0}$ and its first derivative at $\lambda_{0}$. We go one step further and force the second derivative to be null too:

$\left\{\begin{array}{rl}c_{0}\left(\lambda_{0}\right) & =0 \\ \frac{\partial c_{0}}{\partial \lambda}\left(\lambda_{0}\right) & =0 \\ \frac{\partial^{2} c_{0}}{\partial \lambda^{2}}\left(\lambda_{0}\right) & =0\end{array}\right.$.

Using the following expressions for the derivatives,

$$
\left\{\begin{aligned}
\frac{\partial c_{0}}{\partial \lambda}(\lambda) & =-\frac{i \lambda_{0}}{2 \pi \lambda^{2}} \int_{0}^{\pi} G_{0}(\theta) e^{i \lambda_{0} G_{0}(\theta) / \lambda} \mathrm{d} \theta \\
\frac{\partial^{2} c_{0}}{\partial \lambda^{2}}(\lambda) & =\frac{i \lambda_{0}}{\pi \lambda^{3}} \int_{0}^{\pi}\left[G_{0}(\theta)+\frac{i \lambda_{0} G_{0}^{2}(\theta)}{2 \lambda}\right] e^{i \lambda_{0} G_{0}(\theta) / \lambda} \mathrm{d} \theta,
\end{aligned}\right.
$$

one can rewrite Eq. (3):

$$
\begin{array}{r}
\int_{0}^{\pi} \exp \left(i G_{0}(\theta)\right) \mathrm{d} \theta=0 \\
\int_{0}^{\pi} G_{0}(\theta) \exp \left(i G_{0}(\theta)\right) \mathrm{d} \theta=0 \\
\int_{0}^{\pi} G_{0}^{2}(\theta) \exp \left(i G_{0}(\theta)\right) \mathrm{d} \theta=0 .
\end{array}
$$

One family of $G_{0}$ functions that obey Eq. (5a) is defined by

$\forall \theta \in\left[\frac{\pi}{2}, \pi\left[, \exists \theta_{1} \in\left[0, \frac{\pi}{2}\left[, G_{0}(\theta)=G_{0}\left(\theta_{1}\right)+\left(2 k_{\theta}+1\right) \pi\right.\right.\right.\right.$,

with $k_{\theta}$ in $\mathbb{Z}$. The four quadrant phase mask (FQPM, Rouan et al. 2000) obeys this equation with $k_{\theta}=0$ and $G_{0}(\theta)=0$ for $\theta \in$ $[0, \pi / 2[$. The eight octant (EOPM, Murakami et al. 2008) and the six level phase masks (SLPM, Hou et al. 2014) are also part of the family if we allow $k_{\theta}$ to vary with $\theta\left(k_{\theta}=-1\right.$ or $\left.k_{\theta}=0\right)$.

We consider the family of functions defined by condition (6) with $k_{\theta}=k$ not varying with $\theta$. We then search for $G_{0}$ functions that also obey Eq. (5b) that can be written

$$
\int_{0}^{\pi / 2} G_{0}(\theta) \exp \left(i G_{0}(\theta)\right) \mathrm{d} \theta+\int_{\pi / 2}^{\pi} G_{0}(\theta) \exp \left(i G_{0}(\theta)\right) \mathrm{d} \theta=0 .
$$

We account for condition (6) and simplify the result to:

$(2 k+1) \pi \int_{0}^{\pi / 2} \exp \left(i G_{0}(\theta)\right) \mathrm{d} \theta=0$.

This equation is validated by the family of functions such as

$\forall \theta \in\left[\frac{\pi}{4}, \frac{\pi}{2}\left[, \exists \theta_{1} \in\left[0, \frac{\pi}{4}\left[, G_{0}(\theta)=G_{0}\left(\theta_{1}\right)+\left(2 p_{\theta}+1\right) \pi\right.\right.\right.\right.$,

with $p_{\theta}$ in $\mathbb{Z}$. Unlike the FQPM and the SLPM, the EOPM obeys this condition with $p_{\theta}=0$ and $G_{0}(\theta)=0$ for $\theta \in[0, \pi / 2[$.

Finally, we search for a sub-family that also obeys Eq. (5c) imposing a uniform $p_{\theta}=p$. One can transform Eq. (5c) into

$$
\begin{aligned}
& \int_{0}^{\pi / 4} G_{0}^{2}(\theta) \exp \left(i G_{0}(\theta)\right) \mathrm{d} \theta \\
& \quad-\int_{0}^{\pi / 4}\left[G_{0}(\theta)+(2 p+1) \pi\right]^{2} \exp \left(i G_{0}(\theta)\right) \mathrm{d} \theta \\
& \quad-\int_{0}^{\pi / 4}\left[G_{0}(\theta)+(2 k+1) \pi\right]^{2} \exp \left(i G_{0}(\theta)\right) \mathrm{d} \theta \\
& \quad+\int_{0}^{\pi / 4}\left[G_{0}(\theta)+(2 k+2 p+2) \pi\right]^{2} \exp \left(i G_{0}(\theta)\right) \mathrm{d} \theta \\
& =0
\end{aligned}
$$

Using Eqs. (5a) and (5b) and conditions (6) and (7), one finds

$$
(4 k p+2 p+2 k+1) \int_{0}^{\pi / 4} \exp \left(i G_{0}(\theta)\right) \mathrm{d} \theta=0
$$

This equation is validated if and only if the integral is null and one family of solutions is

$$
\forall \theta \in\left[\frac{\pi}{8}, \frac{\pi}{4}\left[, \exists \theta_{1} \in\left[0, \frac{\pi}{8}\left[, G_{0}(\theta)=G_{0}\left(\theta_{1}\right)+\left(2 m_{\theta}+1\right) \pi,\right.\right.\right.\right.
$$

with $m_{\theta}$ in $\mathbb{Z}$. This parameter can depend on $\theta$ although we do not use this property in the rest of the paper (we write $m_{\theta}=m$ ). We note that the EOPM does not validate Eq. (8).

Finally, $G_{0}$ functions that obey the three conditions (6)-(8) also obey condition (3) meaning that the coronagraphic performance is perfect at the optimized wavelength $\lambda_{0}\left(c_{0}\left(\lambda_{0}\right)=0\right)$ and is not very sensitive to chromatism (the two first derivatives of $c_{0}$ are null at $\lambda_{0}$ ). For example, given $f$, a function defined over [0, $\pi / 8$ [ and $m, p$, and $k$, three numbers in $\mathbb{Z}$, one family of solutions is

$$
G_{0}(\theta)=\left\{\begin{array}{lc}
f(\theta) & 0 \leq \theta<\frac{\pi}{8} \\
f\left(\theta-\frac{\pi}{8}\right)+[2 m+1] \pi & \frac{\pi}{8} \leq \theta<\frac{\pi}{4} \\
f\left(\theta-\frac{\pi}{4}\right)+[2 p+2 m+2] \pi & \frac{\pi}{4} \leq \theta<\frac{3 \pi}{8} \\
f\left(\theta-\frac{3 \pi}{8}\right)+[2 p+1] \pi & \frac{3 \pi}{8} \leq \theta<\frac{\pi}{2} \\
f\left(\theta-\frac{\pi}{2}\right)+[2 k+2 m+2] \pi & \frac{\pi}{2} \leq \theta<\frac{5 \pi}{8} \\
f\left(\theta-\frac{5 \pi}{8}\right)+[2 k+1] \pi & \frac{5 \pi}{8} \leq \theta<\frac{3 \pi}{4} \\
f\left(\theta-\frac{3 \pi}{4}\right)+[2 k+2 p+2] \pi & \frac{3 \pi}{4} \leq \theta<\frac{7 \pi}{8} \\
f\left(\theta-\frac{7 \pi}{8}\right)+[2 k+2 p+2 m+3] \pi & \frac{7 \pi}{8} \leq \theta<\pi \\
G_{0}(\theta-\pi) & \pi \leq \theta<2 \pi .
\end{array}\right.
$$

We note that we can reorder the values $G_{0}(\theta)$ inside each interval $[n \pi / 8,(n+1) \pi / 8[$ with $n$ in $\llbracket 0,7 \rrbracket$ as long as conditions (6)-(8) are validated.

\subsection{Wrapped vortex phase mask}

Equation (9) defines an infinite number of functions. Here, we use an additional condition: avoiding $\pi$ discontinuities. Such $\pi$ transitions that exist in the FQPM, SLPM, and EOPM strongly affect the image of off-axis sources reducing the effective field of view. One example of $G_{0}$ that obeys Eq. (9) with no $\pi$ discontinuities is defined by $m=0, p=0, k=-1$ and $f(\theta)=8 \theta$ :

$G_{0}(\theta)=\left\{\begin{array}{lc}8 \theta & 0 \leq \theta<\frac{3 \pi}{8} \\ 8 \theta-2 \pi & \frac{3 \pi}{8} \leq \theta<\frac{\pi}{2} \\ 8 \theta-4 \pi & \frac{\pi}{2} \leq \theta<\frac{5 \pi}{8} \\ 8 \theta-6 \pi & \frac{5 \pi}{8} \leq \theta<\pi \\ G_{0}(\theta-\pi) & \pi \leq \theta<2 \pi\end{array}\right.$

This function is continuous everywhere except at positions $\theta=$ $0,3 \pi / 8, \pi / 2,5 \pi / 8, \pi, 11 \pi / 8,3 \pi / 2,13 \pi / 8$ where there are $2 \pi$ steps (Figs. 2 and 3). The resulting phase mask that we refer to as the wrapped vortex of topological charge 8 is a phase ramp that would go from 0 to $16 \pi$ but that is wrapped eight times.

Replacing $f(\theta)=8 \theta$ by $f(\theta)=0$, Eq. (10) describes one mask derived from Diophantine optics (Fig. 3 in Rouan 2016). In future work, we will study how Diophantine optics can be linked to the family of functions described by Eq. (9). 


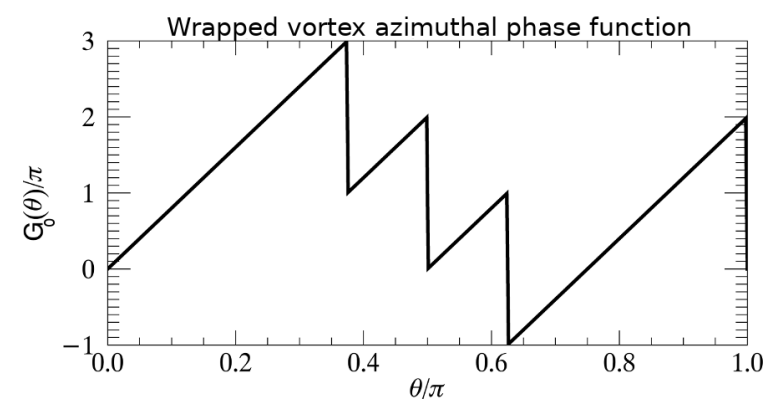

Fig. 2. Wrapped vortex ramp $G_{0}$ defined by Eq. (10).

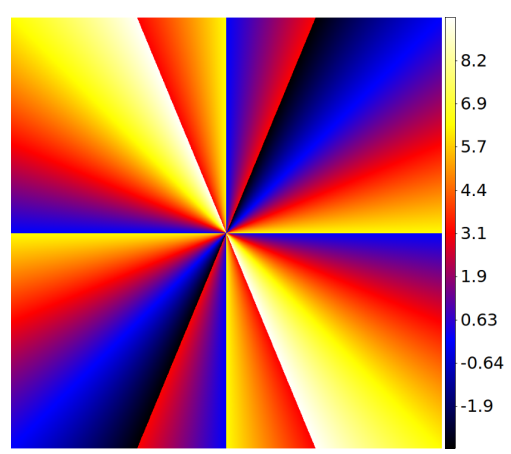

Fig. 3. Two-dimensional map of the wrapped vortex defined by Eq. (10). The color bar gives the phase in radians.

\section{Numerical simulations of a wrapped vortex}

\subsection{Coronagraphic attenuation}

We run numerical simulations to estimate the coronagraphic attenuation of the central star using the wrapped vortex defined by Eq. (10). We assume Fourier optics and use fast Fourier transforms to propagate light from a pupil plane to a focal plane and vice versa. The entrance pupil is a circular disk of 256 pixels in arrays of 16384 pixels. The phase mask is set in the first focal plane. The Lyot diaphragm is in the following pupil plane. Eventually, the image is recorded in the last focal plane.

We assume the phase mask is made of a single piece of material that is etched to induce the perfect phase shift $G_{0}$ at the optimized wavelength $\lambda_{0}$. We neglect the variation of the optical index with wavelength (see Sect. 2.2). We assume a Lyot stop whose diameter $D$ is $95 \%$ of the entrance pupil diameter. For each wavelength, we calculate the integrated energy inside $1 \lambda / D$-diameter at the center of the coronagraphic image. We normalize this energy by the energy that is calculated in the same way using the noncoronagraphic image (i.e., $G_{0}(\theta)=0$ everywhere) at the same wavelength. The result is the attenuation $A$ that is plotted in Fig. 4. By definition, $A$ is equal to 1 at all wavelengths if no coronagraph is used. We compare the wrapped vortex to FQPM, the vortex phase mask of topological charge 8, and SLPM. All masks are optimized at $\lambda_{0}=700 \mathrm{~nm}$.

As expected from Hou et al. (2014) and as demonstrated in laboratory by Patru et al. (2018), SLPM is less sensitive to chromatism than FQPM. The attenuation for the vortex of charge 8 is minimum at $\lambda_{0}, \lambda_{1}=16 / 18 \lambda_{0}=622 \mathrm{~nm}$ (equivalent to a vortex of charge 9) and at $\lambda_{2}=16 / 14 \lambda_{0}=800 \mathrm{~nm}$ (equivalent to a vortex of charge 7). The attenuations at $\lambda_{1}$ and $\lambda_{2}$ are not infinite because the charge is odd. They are large however because the charge is high (Mawet et al. 2005). The wrapped vortex attenuates the starlight up to approximately 10 times better than the SLPM and up to approximately 500 times better than

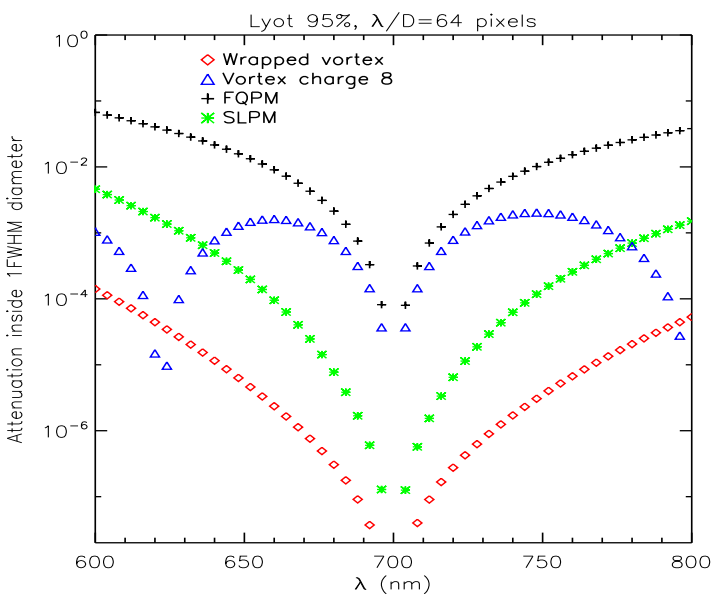

Fig. 4. Numerical simulations: Coronagraphic attenuation integrated inside a disk of $1 \lambda / D$-diameter centered on the optical axis for a four quadrant phase mask (black plus), a vortex phase mask of topological charge 8 (blue triangle), a six-level phase mask (green stars), and a wrapped-vortex phase mask defined by Eq. (10) (red diamond).

the FQPM over the $\sim 29 \%$ bandpass (600 to $800 \mathrm{~nm}$ ). Except for short bandpasses around $\lambda_{1}$ and $\lambda_{2}$, the wrapped vortex attenuation is also 10 to 100 times better than for the vortex of charge 8 . Finally, on average, the wrapped vortex attenuates the starlight by a factor of $10^{4}$ over a $29 \%$ bandpass, $10^{5}$ over a $18 \%$ bandpass, and $10^{6}$ over a $10 \%$ bandpass.

\subsection{Off-axis transmission}

In Sect. 3.1, we show that the wrapped vortex phase mask can attenuate the central starlight over a large bandpass. The second role of a coronagraph is to maximize the transmission of the planet light through the instrument. Therefore, we study the transmission of the coronagraph for an off-axis source (e.g., the planet). The transmission is the ratio between the maximum of the image of off-axis sources obtained with the phase mask and without the mask (i.e., $G_{0}(\theta)=0$ ). We calculate the transmission at several wavelengths $\lambda$ for sources angularly separated from $-30 \lambda / D$ to $30 \lambda / D$ from the optical axis with steps of $1 \lambda / D$. Maps are shown in Fig. 5 for five wavelengths from 600 to $800 \mathrm{~nm}$. At $700 \mathrm{~nm}$, the map is centrosymmetrical because the steps of material on the phase mask induce $2 \pi$ phase shifts. At this wavelength, the inner working angle at which $50 \%$ of the planet flux is transmitted is $\sim 4 \lambda / D$. If the wavelength is not equal to $700 \mathrm{~nm}$, the steps of materials induce phase shifts that are not $2 \pi$ and interference modifies the transmitted flux. Therefore, the transmission map is not centrosymmetrical. Such inhomogeneous transmission over the field of view requires careful calibrations to extract the photometry of a planet or of a circumstellar disk. However, we measure that over a $100 \mathrm{~nm}$ bandpass around $\lambda_{0}=700 \mathrm{~nm}$ (14\% bandpass), the transmission varies by less than $10 \%$.

In Fig. 6, we plot the azimuthal average of the energy transmission for a point-like source at 639,705 , and $783 \mathrm{~nm}$ as a function of the angular separation from the optical axis for a wrapped vortex phase mask optimized at $700 \mathrm{~nm}$. The three wavelengths are the ones of the laser diodes that we use in the experiment (see Sect. 5.3). The error bars show the standard deviation computed azimuthally. We find that the transmission curves are very similar with an inner working angle of $\sim 5 \lambda / D$. For comparison, we plot in Fig. 7 the same curve at $705 \mathrm{~nm}$ for 


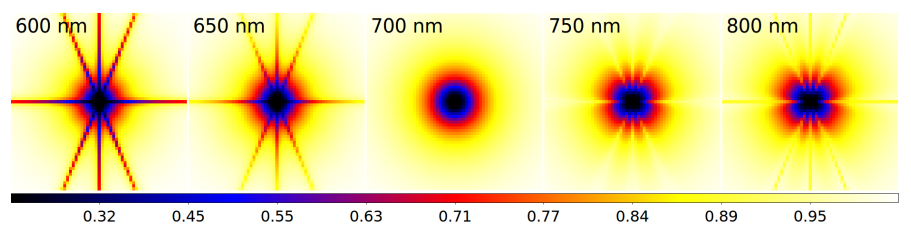

Fig. 5. Numerical simulations: Maps of the energy transmission for a point-like source observed through a coronagraph using a wrapped vortex phase mask at wavelengths $\lambda$ from 600 to $800 \mathrm{~nm}$. The excursion of the source goes from $-30 \lambda / D$ to $30 \lambda / D$ from the center of the phase mask in both horizontal and vertical directions. The color bar goes from 0 (no light is transmitted) to 1 (all flux is transmitted).

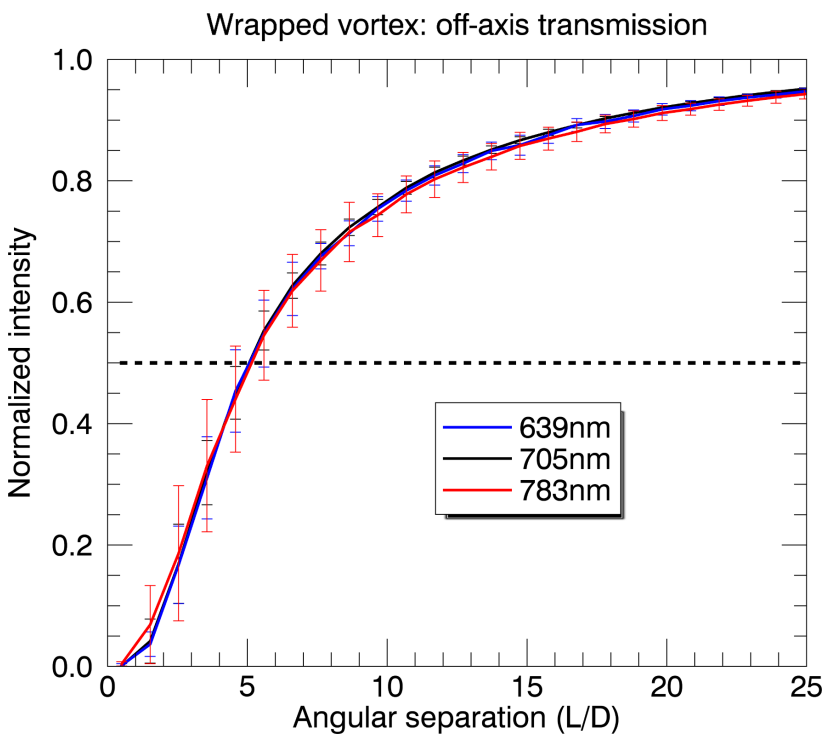

Fig. 6. Numerical simulations: Azimuthal average of the energy transmission for a point-like source as a function of the angular separation from the optical axis for a wrapped vortex phase mask optimized at $700 \mathrm{~nm}$. The transmissions are calculated at $639 \mathrm{~nm}$ (blue), $705 \mathrm{~nm}$ (black), and $783 \mathrm{~nm}$ (red). The error bars show the standard deviation computed azimuthally.

phase masks made of a single piece of material: FQPM, SLPM, vortex of charge 8 , and wrapped vortex. The masks are optimized at $700 \mathrm{~nm}$. As already demonstrated in Patru et al. (2018), SLPM and FQPM transmission curves are the same with an inner working angle of $\sim 2 \lambda / D$. The large error bar at $2 \lambda / D$ comes from the strong variation of the transmission in the field of view because of the $\pi$-transitions of these masks. We also find that the transmission curves are the same for the wrapped vortex and the vortex of charge 8 with a $\sim 5 \lambda / D$ inner working angle. However, unlike the wrapped vortex, we find from numerical simulations (not plotted here) that the transmission curve slightly evolves with wavelength for the scalar vortex of charge 8 .

\section{Manufacturing of wrapped vortex mask}

\subsection{Specifications}

We ordered one wrapped vortex component from the Zeiss company whose phase function is described by Eq. (10). The component is a square of $17.5_{-0.1}^{+0} \mathrm{~mm} \times 17.5_{-0.1}^{+0} \mathrm{~mm}$ made of fused silica with a thickness of $1 \mathrm{~mm}$. The parallelism between the two faces of the substrate is smaller than 20 arcsec. The surface quality before ion-etching is better than $\lambda / 10$ peak-tovalley at $633 \mathrm{~nm}$ in transmission for spatial frequencies larger

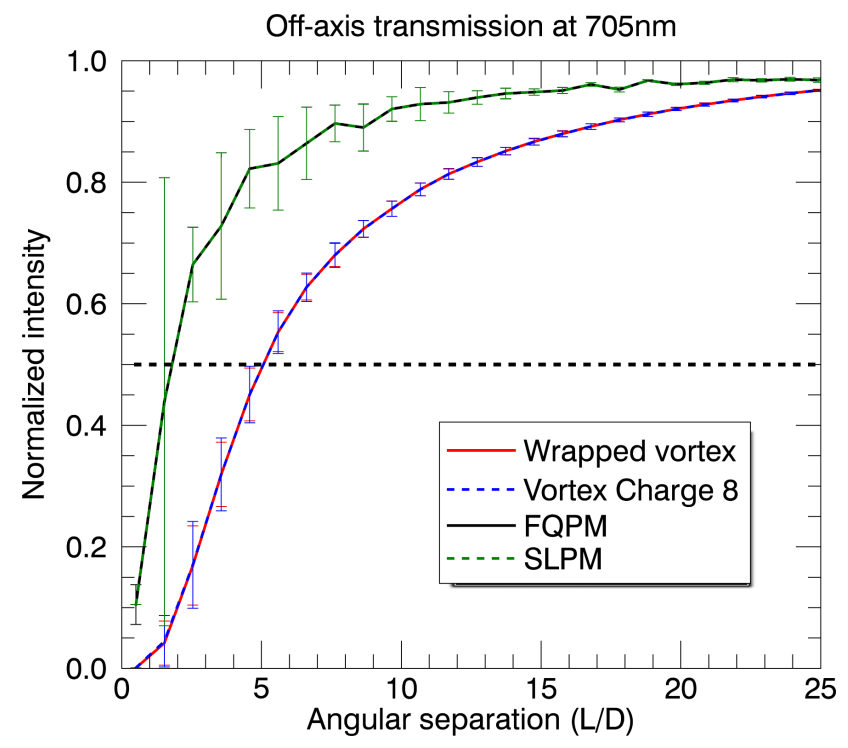

Fig. 7. Numerical simulations: Same as Fig. 6 at $705 \mathrm{~nm}$ but for several phase masks optimized at $700 \mathrm{~nm}$.

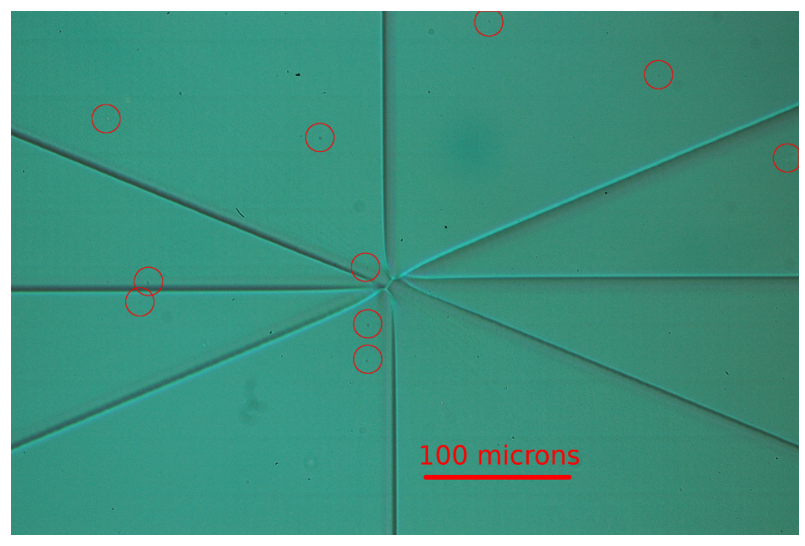

Fig. 8. Image of the wrapped vortex component obtained with an optical microscope. Dusts and defects on the component are encircled in red. The other defects are on the microscope optics.

than $0.05 \mathrm{cy} \mathrm{mm}^{-1}$. The wrapped ramp of material thickness is calculated so that the phase function obeys Eq. (10) with an accuracy of $\pm 5 \%$ at the optimized wavelength of $700 \mathrm{~nm}$. It was created by ion-etching - the manufacturer can etch continuous $2 \mathrm{D}$ functions. After the ramp is etched, both faces are coated so that the average reflection coefficient in amplitude is below $0.2 \%$ per surface between 600 and $800 \mathrm{~nm}$.

\subsection{Delivered component}

All specifications are respected by the manufacturer but the optimized wavelength is larger than expected. This is because we asked the manufacturer to fabricate different phase masks on the same wafer. Therefore, it was complicated to constrain the optimal depth of etching and the different 2D phase functions simultaneously. Confocal measurements provided by the manufacturer find that the step of material near the border of the mask (meaning at $\sim 8.7 \mathrm{~mm}$ from the center) is about $10 \%$ higher than the specification. Assuming the step of material has the same height at the center of the mask, we can expect the mask to be optimized at $770 \mathrm{~nm}$ instead of the specified $700 \mathrm{~nm}$. Figure 8 shows a visible microscope image of the component. Most of 


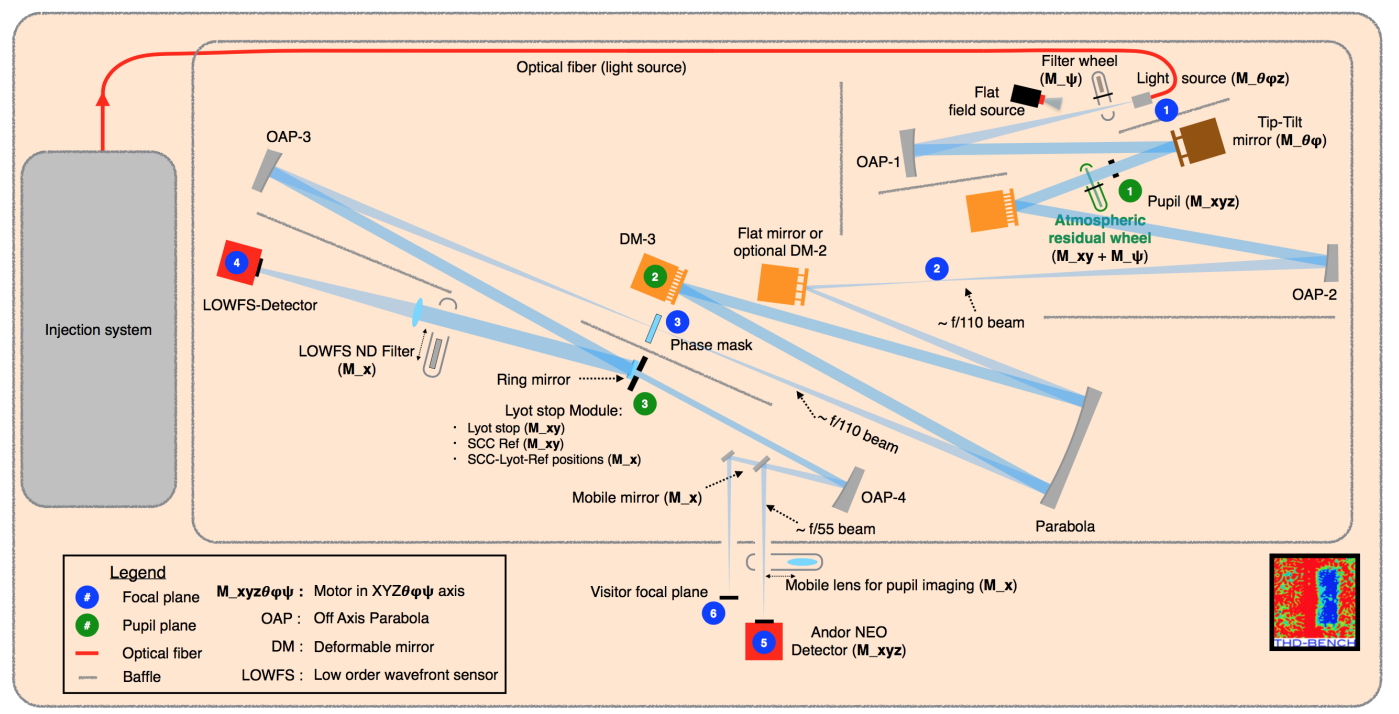

Fig. 9. Layout of the THD2 bench. Active optics are in orange. Blue disks mark focal planes and green disks pupil planes.

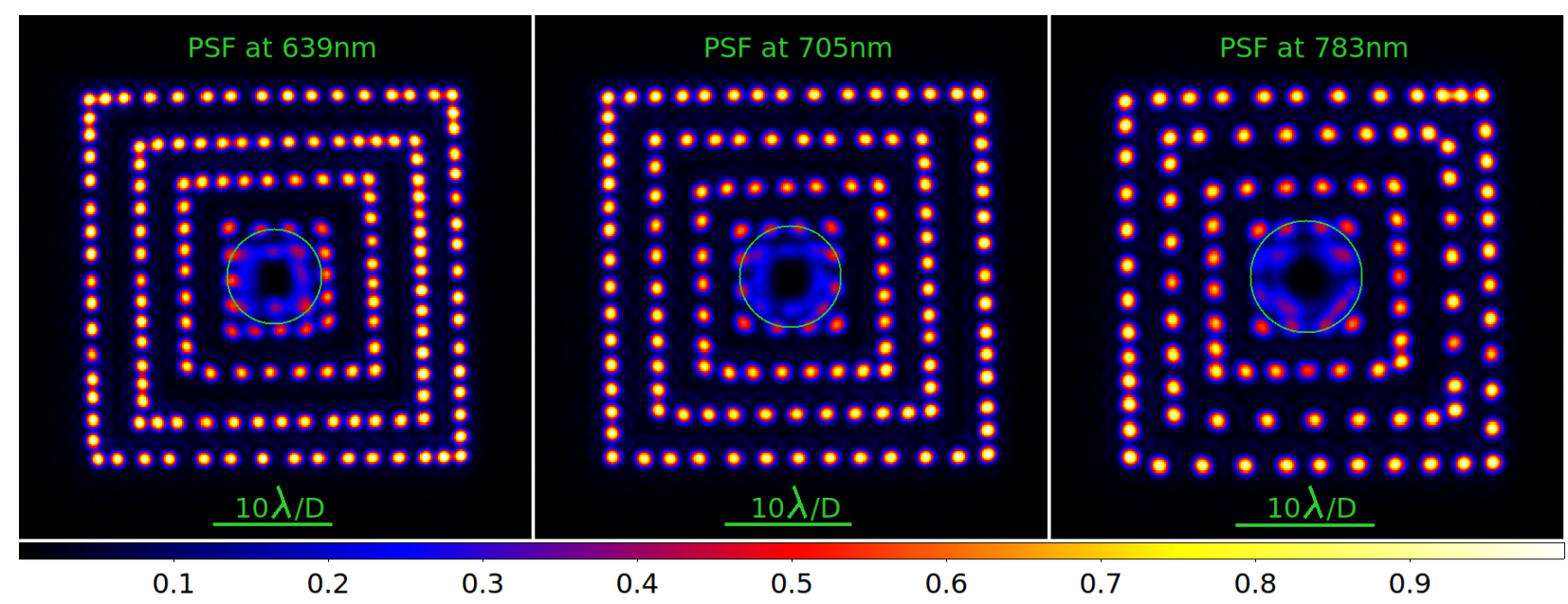

Fig. 10. Laboratory measurement: Superposition of the images of off-axis sources at $639 \mathrm{~nm}($ left), $705 \mathrm{~nm}$ (middle), and $783 \mathrm{~nm}$ (right). The color bar gives the transmission through the wrapped vortex coronagraph. Circles have a radius of $4 \lambda / D$ from the optical axis.

the detected spots are defects on the microscope optics. Only a little dust and a few defects are present on the component (encircled in red). In the following sections, the diameter of the point spread function that is focused on the phase mask is between 60 and $100 \mu \mathrm{m}$ depending on the wavelength. Therefore, only the three defects that are the closest to the center of the mask are lit up. As they are very small in size $(<0.4 \mu \mathrm{m})$, we expect their effect to be negligible. From the image, we also measure the width of the $2 \pi$-phase steps to be between approximately 5 and $8 \mu \mathrm{m}$. This is quite large with respect to what can be fabricated for binary masks like FQPM $(<0.5 \mu \mathrm{m}$ in Bonafous et al. 2016). However, unlike the FQPM, numerical simulations predict that the wrapped vortex gives good coronagraphic attenuations even with transition widths of $\sim 10 \%$ of $\lambda / D$. We believe that this is because the phase steps are $2 \pi$ in phase instead of $\pi$ for the FQPM.

\section{Laboratory performance of a wrapped vortex}

\subsection{THD2 bench}

We probe the coronagraphic performance of the component described in Sect. 4 on the THD2 bench that has already been described in the literature (Baudoz et al. 2018a,b; Patru et al. 2018; Potier et al. 2018; Singh et al. 2019). Figure 9 shows the layout of the bench. In this paper, we removed the atmospheric residual wheel from the beam so that we simulate a space-like telescope with an unobscured pupil of $8.23 \mathrm{~mm}$ diameter. A linear polarizer is set in front of the Andor NEO detector. A flat mirror replaces the deformable mirror DM2. The wrapped vortex phase mask is set in focal plane 3 (blue disk). The low-order wavefront sensor (LOWFS, Singh et al. 2014) is used to stabilize the centering of the beam onto the phase mask. Deformable mirrors, namely DM3 set in a pupil plane and DM1 at $26.9 \mathrm{~cm}$ from the pupil plane, are used to control both phase and amplitude aberrations of the wavefront and thereby minimize the speckle intensity in a $360^{\circ}$ field of view around the optical axis (i.e., the star center). The self-coherent camera is used for focal plane wavefront sensing and to control the deformable mirrors (Galicher et al. 2010; Mazoyer et al. 2013a,b, 2014). Several sources of the injection system can light up the bench: laser diodes $(638.6 \pm 2.3 \mathrm{~nm}, 704.5 \pm 2.1 \mathrm{~nm}$ and, $783 \pm 2.3 \mathrm{~nm})$ or the supercontinuum source associated with spectral filters with a $\sim 10 \mathrm{~nm}$ bandwidth. The flux and the spectrum of the light that enters the THD2 bench are continuously measured so that the photometry can easily be calibrated in the recorded images. 

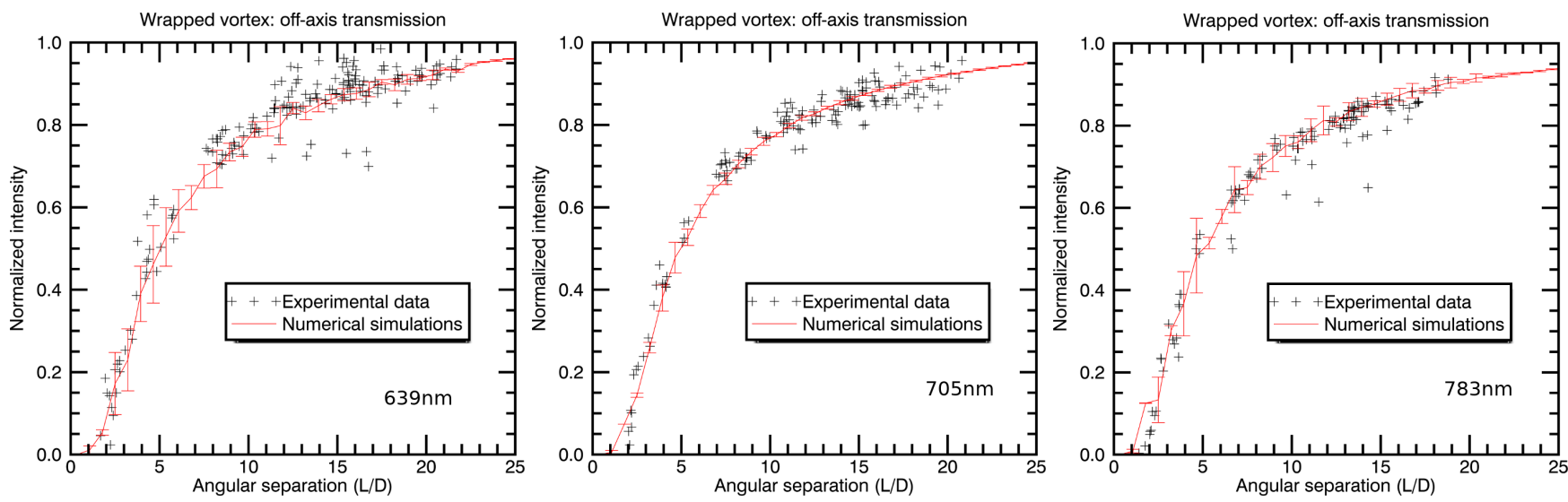

Fig. 11. Laboratory measurement: Transmission of an off-axis source through the wrapped vortex coronagraph against the angular separation at three wavelengths: $639 \mathrm{~nm}$ (left), $705 \mathrm{~nm}$ (middle), and $783 \mathrm{~nm}($ right). Predictions from numerical simulations are plotted in red.

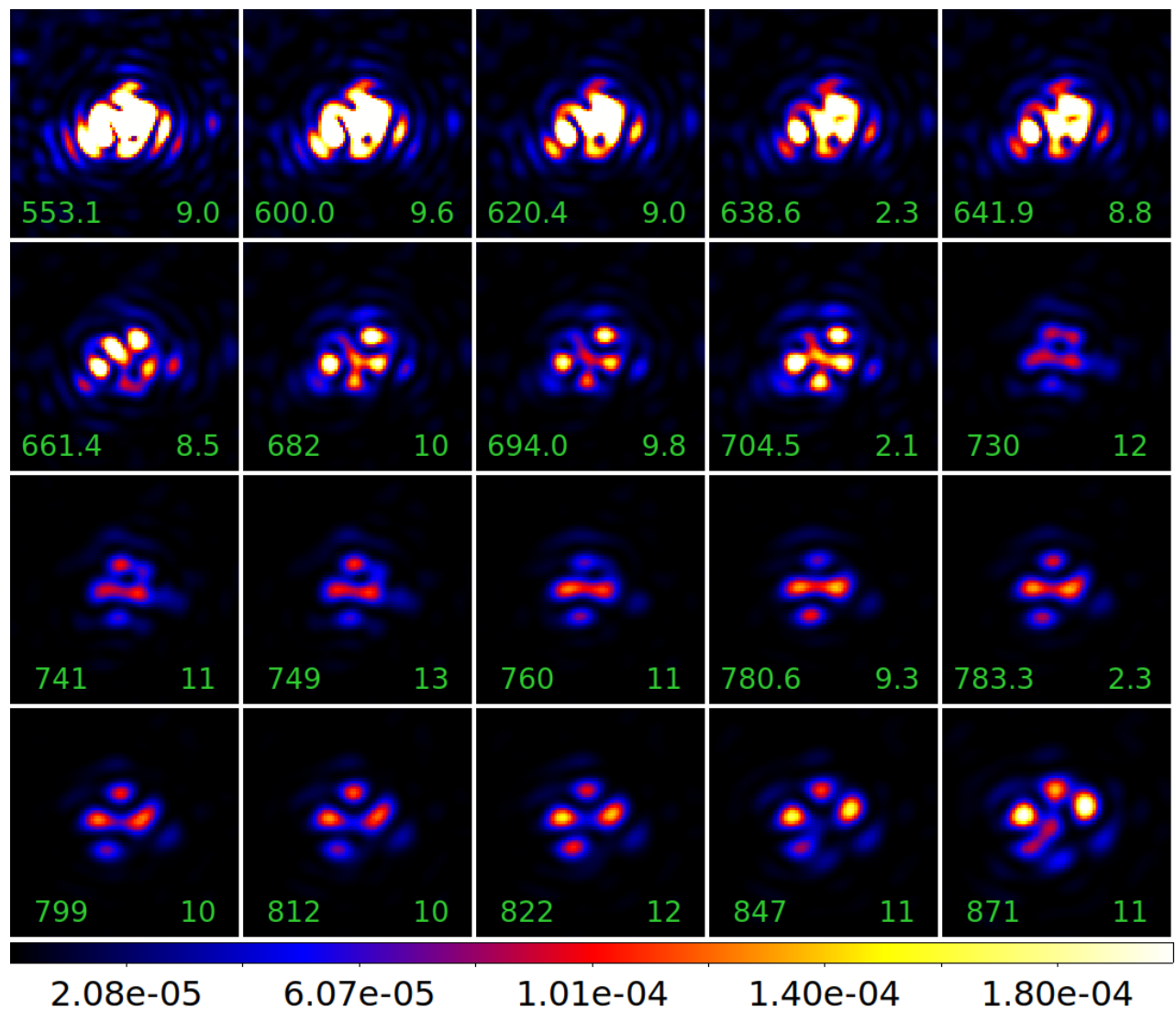

Fig. 12. Laboratory measurement: Coronagraphic images recorded at different wavelengths (central wavelength in the bottom left and spectral bandwidth in the bottom right of each image). Speckles are minimized using the laser diode at $783 \mathrm{~nm}$. The image size is $12 \lambda / D \times 12 \lambda / D$ at $704.5 \mathrm{~nm}$. The color bar gives the intensity normalized by the maximum of the noncoronagraphic image.

\subsection{Transmission of an off-axis source}

In this section, we study the transmission of the coronagraph for an off-axis source like an exoplanet, as done by numerical simulations in Sect. 3.2. We record off-axis images at the three laser diode wavelengths moving the beam in the field thanks to the tip-tilt mirror (dark brown in Fig. 9). The Lyot stop diameter is $8.00 \mathrm{~mm}$ ( $97 \%$ of the entrance pupil diameter). At maximum, we can move the source to $\sim 22 \lambda / D, \sim 21 \lambda / D$, and $\sim 19 \lambda / D$ from the optical axis at $639 \mathrm{~nm}, 705 \mathrm{~nm}$, and $783 \mathrm{~nm}$ respectively. From numerical simulations, we find the transmission of the coronagraph at these separations is theoretically $\sim 93.4 \%$, $\sim 92.9 \%$, and, $90.5 \%$ respectively. Therefore, for each wavelength, we select the five farthest images. We calculate the average $s$ of their separations to the optical axis (the five separations are within $1 \lambda / D$ of each other). We then normalize all the images so that the transmission at $s$ is equal to the transmission found by numerical simulations in Sect. 3.2. Doing so, we suppose the transmission of the fused silica that the mask is made of is $100 \%$. This is a reasonable assumption because the coating is of high quality (Sect.4.1). Figure 10 shows the superposition of the normalized images of all the off-axis sources for the three wavelengths. The experimental transmissions of the wrapped vortex coronagraph (i.e. the maximum of each off-axis 


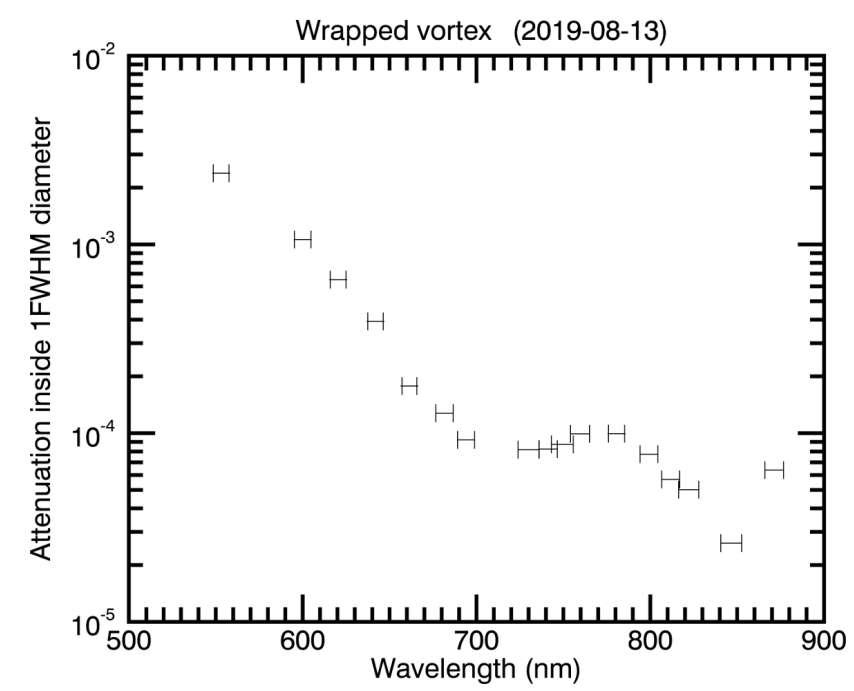

Fig. 13. Laboratory measurement: Coronagraphic attenuation $A$ integrated inside a disk of $1 \lambda / D$-diameter centered on the optical axis. The horizontal excursions give the filter bandwidth.

image) are then plotted as a function of the angular separation in Fig. 11. The curves obtained by numerical simulations are overplotted in red. Curves and experimental data are in good agreement at the positions used for the normalization of the data (the farthest separations from the star). They are also consistent at all angular separations, validating the results of our numerical simulations. In conclusion, we find that the inner working angle at which the transmission of an off-axis source reaches $50 \%$ is between $4 \lambda / D$ and $6 \lambda / D$ for the wrapped vortex phase mask.

\subsection{Coronagraphic attenuation}

In the second laboratory experiment, we measure the coronagraphic attenuation of the star image as done by numerical simulations in Sect. 3.1. The Lyot stop diameter is $7.90 \mathrm{~mm}(96 \%$ of the entrance pupil diameter). We first minimize the speckle intensity at $783 \mathrm{~nm}$ (laser diode) in a $27 \lambda / D \times 27 \lambda / D$ square centered on the optical axis: we measure the electric field using the self-coherent camera and we use both deformable mirrors DM1 and DM3 to compensate for phase and amplitude aberrations. The deformable mirror shapes are then fixed and we light up the system at different wavelengths. Finally, we record coronagraphic images and noncoronagraphic images for photometry calibration. Noncoronagraphic images are recorded in the presence of the wrapped vortex phase mask but at $\sim 20 \lambda / D$ from the center of the mask. We show in Sect. 5.2 that the transmission of the coronagraph at such separations is below 95\%. Therefore, we use the transmission curve calculated by numerical simulations to calibrate the noncoronagraphic images.

Figure 12 shows the coronagraphic images. The central wavelength and the spectral bandwidth of the filter (or the laser diode) are written in the bottom left and right of each image respectively. The color bar gives the intensity normalized by the maximum of the noncoronagraphic image. The images show the same speckle pattern between $\sim 680$ and $870 \mathrm{~nm}$. Below $680 \mathrm{~nm}$, images slightly evolve and the attenuation degrades. This is also visible in Fig. 13, which plots the coronagraphic attenuation $A$ defined in Sect. 3.1 as a function of wavelength. The horizontal excursions give the filter bandwidth. The attenuation is better than $10^{-4}$ at least between $680 \mathrm{~nm}$ and $870 \mathrm{mn}$ corresponding to a $>25 \%$ bandpass. We could not measure the performance at wavelengths larger than $870 \mathrm{~nm}$ which is the current cut-off of the injection system. This result is consistent with the numerical simulations that predict an attenuation better than $10^{-4}$ over a $29 \%$ bandpass (Fig. 4) and with the expected central wavelength of $\sim 770 \mathrm{~nm}$ (Sect. 4.2). The point at $850 \mathrm{~nm}$ is lower than its neighbors although there are bright speckles in the image (Fig. 12). The speckles are further away than $0.5 \lambda / D$ from the center because of spectral dispersion.

\subsection{Coronagraphic dark holes}

The third experiment is an analysis of the ability to reach very high contrast at several wavelengths using a wrapped vortex phase mask. In an optimal protocol, one would optimize the DM1 and DM3 deformable mirror shapes at a central wavelength to create a dark hole (i.e., region of high contrast in the coronagraphic image). One would then light the bench up with several sources to record dark hole images at all wavelengths. Using the wrapped vortex phase mask and another broadband coronagraph - vector vortex plus polarizers - we recently noticed that there is a slow evolution of the speckle pattern at mid-spatial frequencies. Therefore, there are small chromatic aberrations on the current bench and we are still investigating the reasons for this evolution.

Because of these aberrations, we have to slightly modify the deformable mirror shapes to minimize the speckle intensity at each wavelength. Hence, in this section, for each laser diode $(638.6 \pm 2.3 \mathrm{~nm}, 704.5 \pm 2.1 \mathrm{~nm}$ and, $783 \pm 2.3 \mathrm{~nm})$, we independently measure the electric field associated to the stellar speckles using the self-coherent camera, and we minimize the star intensity inside a dark hole. We record coronagraphic images and then noncoronagraphic images moving the source image to $\sim 20 \lambda / D$ from the center of the phase mask. As in Sect. 5.3, we correct the photometry of the noncoronagraphic images from the transmission of the coronagraph that is not exactly $100 \%$ at these separations. Finally, we normalize the coronagraphic images by the maximum of the noncoronagraphic image and correct each pixel by the coronagraph transmission using the curve obtained in numerical simulations (Fig. 11).

Results for a $360^{\circ}$ full dark hole are presented in Sect. 5.4.1. Deeper contrast levels are then obtained when reducing the dark hole size as shown in Sect. 5.4.2. For these measurements, the Lyot stop diameter is $6.50 \mathrm{~mm}$ (79\% of the entrance pupil diameter).

\subsubsection{Full dark hole of $360^{\circ}$}

We first minimize the speckle intensity in a square dark hole of $27 \lambda / D \times 27 \lambda / D$ centered on the optical axis. The coronagraphic images are presented in Fig. 14. The color bar gives the intensity normalized by the maximum of the noncoronagraphic image. As coronagraphic images are corrected by the coronagraph transmission, the color bar indicates the ratio between the flux of the exoplanet that can be detected and the star. In Fig. 15, we plot the $1 \sigma$ contrast curve that is the azimuthal standard deviation in annuli of $0.5 \lambda / D$ for each image of Fig. 14 . The horizontal axis gives the angular separation from the star in $\lambda_{0} / D$ with $\lambda_{0}=705 \mathrm{~nm}$. Inside the dark holes, regions of interest where we would look for exoplanet images, the $3 \sigma$ contrast level is better than $3 \times 10^{-7}$ over a $20 \%$ spectral bandpass at $5-7 \lambda_{0} / D$ or more from the star. 


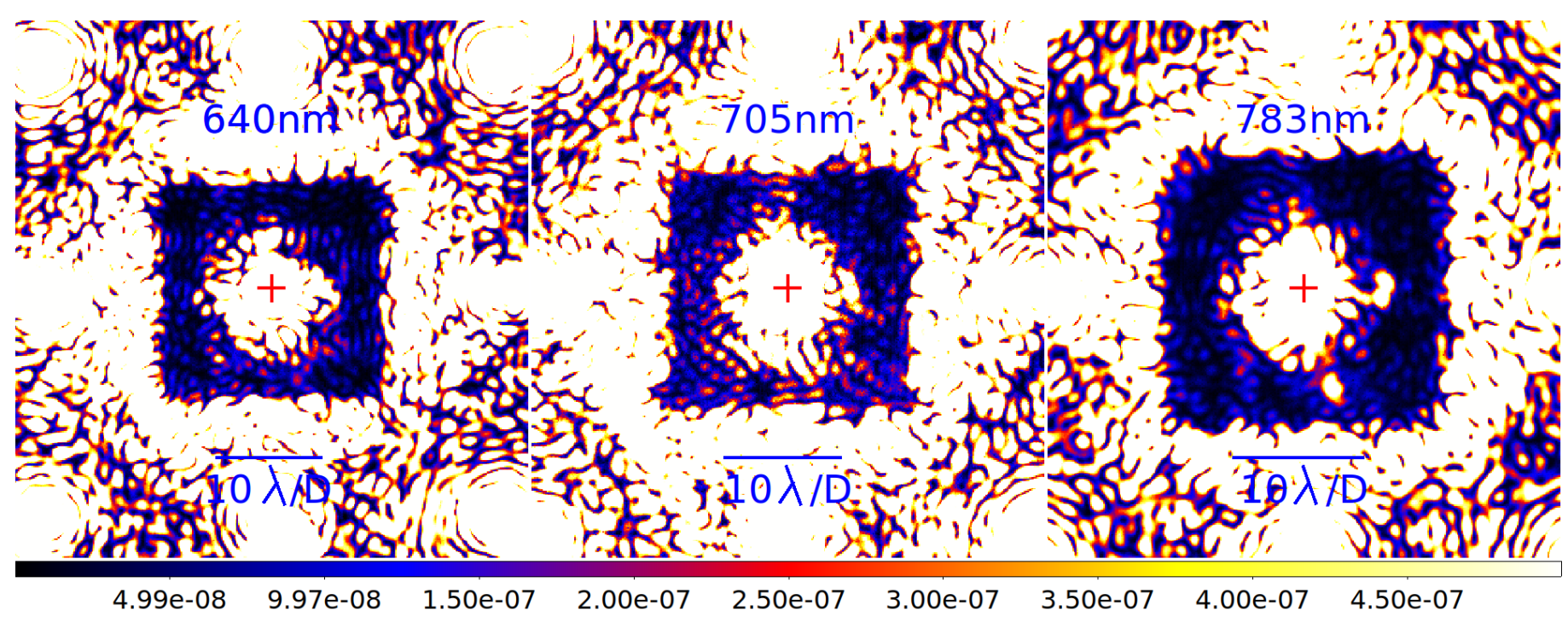

Fig. 14. Laboratory measurement: Coronagraphic images obtained at $639 \mathrm{~nm}($ left $), 705 \mathrm{~nm}($ center $)$, and $783 \mathrm{~nm}($ right $)$ with a $27 \lambda / D \times 27 \lambda / D$ dark hole. The center of the star image is at the center of the field of view (red cross). Each image is corrected by the coronagraph transmission. The color bar gives the intensity normalized by the maximum of the noncoronagraphic image.

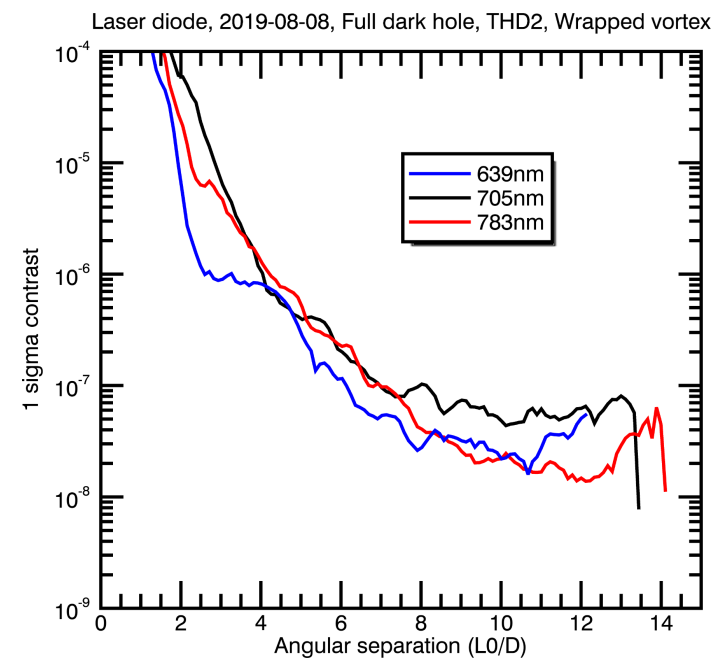

Fig. 15. Laboratory measurement: $1 \sigma$ contrast curves associated with the full dark hole coronagraphic images of Fig. 14 at $639 \mathrm{~nm}$ (blue), $705 \mathrm{~nm}$ (black), and $783 \mathrm{~nm}$ (red). The horizontal axis gives the angular separation from the star in $\lambda_{0} / D$ with $\lambda_{0}=705 \mathrm{~nm}$. These curves are corrected by the coronagraph transmission of an off-axis source (Fig. 11).

\subsubsection{Half-field-of-view dark hole}

In order to reach deeper contrast levels, we reduce the dark hole to a region going from $1 \lambda / D$ to $13.5 \lambda / D$ in one direction and from $-13.5 \lambda / D$ to $13.5 \lambda / D$ in the other direction. Images at the three wavelengths are shown in Fig. 16 and the $1 \sigma$ contrast curves are plotted in Fig. 17. The contrast is plotted against the angular separation expressed in $\lambda_{0} / D$ with $\lambda_{0}=705 \mathrm{~nm}$. At $705 \mathrm{~nm}$, the performance is worse than at the two other wavelengths because of detector noise that is visible in the image. In the $705 \mathrm{~nm}$ image, an exoplanet $10^{7}$ times fainter than its star could be detected at $3 \sigma$ in the raw image (prior to any post-processing of the data). At $639 \mathrm{~nm}$ and $783 \mathrm{~nm}$, an exoplanet up to $\sim 3.10^{7}$ times fainter than its star could be detected at $3 \sigma$ between $7 \lambda_{0} /$ and $12-14 \lambda_{0} / D$. Numerical simulations of such a dark hole using the wrapped vortex phase mask on the THD2 bench predict similar performance with a $3 \sigma$ detection limit at $\sim 3 \times 10^{-8}$ between $6 \lambda_{0} / D$ and $13 \lambda_{0} / D$. In conclusion, the laboratory performance demonstrates the efficiency of the wrapped vortex coronagraph over broadband of at least $20 \%$.

\section{Conclusions}

We used a mathematical approach to derive a family of $2 \mathrm{D}$ azimuthal phase functions for broadband coronagraphy. One of the functions is a wrapped vortex that we studied by numerical simulations. We predicted very efficient coronagraphic attenuation over a $30 \%$ spectral bandpass with very high transmission of the exoplanet light and a $360^{\circ}$ field of view. Taking advantage of a new technology for etching continuous $2 \mathrm{D}$ functions, we manufactured a prototype of the wrapped vortex. We demonstrated in the laboratory that it attenuates the starlight by a factor of $10^{4}$ or more over a $>25 \%$ bandpass. Finally, we obtained dark hole images with a $3 \sigma$ detection limit at $\sim 3 \times 10^{-8}$ at $639 \mathrm{~nm}$ and $783 \mathrm{~nm}$. Aside from very efficient broadband coronagraphic performance, the wrapped vortex requires only one scalar focal plane mask followed by a classical binary Lyot stop. Such a simple design is more attractive than other coronagraphs, which need several optics to be aligned and stabilized like the vector vortex that requires the use of polarizers, or multi-stage devices. Moreover, as a scalar phase mask no polarization filtering is needed and $\sim 100 \%$ of the putative exoplanet light is transmitted towards the science detector. One drawback of the wrapped vortex is the inner working angle that is $5 \lambda / D$. Smaller inner working angles can be achieved with single phase masks such as the FQPM or SLPM at the expense of a narrower spectral band. In future work, families of radially and azimuthally varying wrapped functions will be investigated so that they can be used with obscured pupils. 


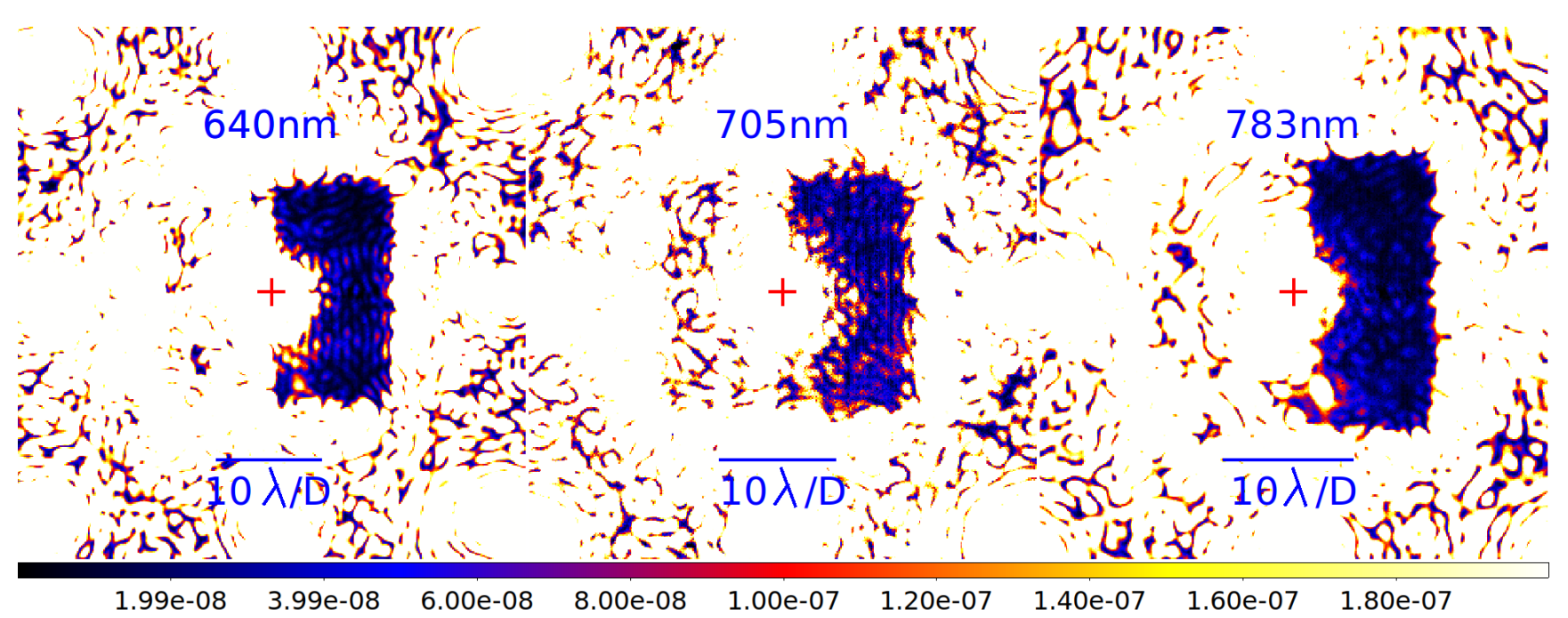

Fig. 16. Laboratory measurement: Coronagraphic images obtained at $639 \mathrm{~nm}($ left), $705 \mathrm{~nm}$ (center), and $783 \mathrm{~nm}($ right) with a half dark hole. The center of the star image is at the center of the field of view (red cross). Each image is corrected by the coronagraph transmission. The color bar gives the intensity normalized by the maximum of the noncoronagraphic image.

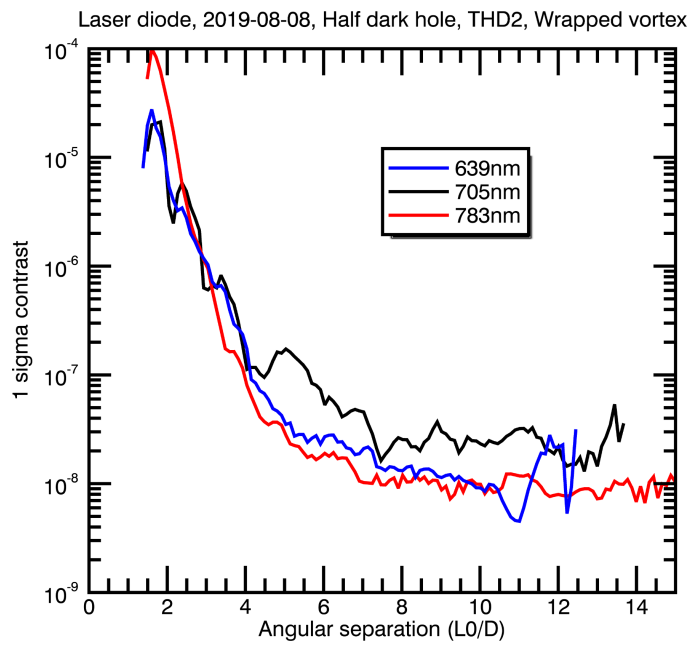

Fig. 17. Laboratory measurement: $1 \sigma$ contrast curves associated with the half dark hole coronagraphic images of Fig. 16 at $639 \mathrm{~nm}$ (blue), $705 \mathrm{~nm}$ (black), and $783 \mathrm{~nm}$ (red). The horizontal axis gives the angular separation from the star in $\lambda_{0} / D$ with $\lambda_{0}=705 \mathrm{~nm}$. These curves are corrected by the coronagraph transmission of an off-axis source (Fig. 11).

Acknowledgements. French co-authors are supported by ANR-14-CE33-0018. Part of this work was supported by the Physics department of the University of Paris.

\section{References}

Adibekyan, V. 2019, Geosciences, 9, 105

Alonso-Floriano, F. J., Sánchez-López, A., Snellen, I. A. G., et al. 2019, A\&A 621, A74

Baba, N., \& Murakami, N. 2003, PASP, 115, 1363

Baudoz, P., Galicher, R., Patru, F., Dupuis, O., \& Thijs, S. 2018a, AO4ELT 2017 conference proceeding [arXiv: 1801.06600 ]

Baudoz, P., Galicher, R., Potier, A., et al. 2018b, in Advances in Optical and Mechanical Technologies for Telescopes and Instrumentation III, SPIE Conf. Ser., 10706, 1070620

Beuzit, J. L., Vigan, A., Mouillet, D., et al. 2019, A\&A, 631, A155

Bonafous, M., Galicher, R., Baudoz, P., Firminy, J., \& Boussaha, F. 2016, Proc. SPIE, 9912, 99126J

Cady, E., Balasubramanian, K., Gersh-Range, J., et al. 2017, Proc. SPIE, 10400, $104000 \mathrm{E}$
Close, L. M., Males, J. R., Morzinski, K. M., et al. 2018, Proc. SPIE, 10703, 107030

D'Angelo, G., \& Lissauer, J. J. 2018, Formation of Giant Planets, 140

Espinoza, N., Rackham, B. V., Jordán, A., et al. 2019, MNRAS, 482, 2065

Galicher, R., Baudoz, P., \& Baudrand, J. 2011, A\&A, 530, A43

Galicher, R., Baudoz, P., Rousset, G., Totems, J., \& Mas, M. 2010, A\&A, 509, A31

Gravity Collaboration (Lacour, S., et al.) 2019, A\&A, 623, L11

Hou, F., Cao, Q., Zhu, M., \& Ma, O. 2014, Optics Express, 22, 1884

Izidoro, A., \& Raymond, S. N. 2018, Formation of Terrestrial Planets, 142

Jovanovic, N., Absil, O., Baudoz, P., et al. 2018, Proc. SPIE, 10703, 107031U

Lozi, J., Guyon, O., Jovanovic, N., et al. 2018, Proc. SPIE, 10703, 1070359

Ma, O., Cao, Q., \& Hou, F. 2012, Opt. Exp., 20, 10933

Macintosh, B., Graham, J. R., Ingraham, P., et al. 2014, Proc. Nat. Academy Sci., 111,12661

Marois, C., Lafrenière, D., Doyon, R., Macintosh, B., \& Nadeau, D. 2006, ApJ, 641,556

Mawet, D., Riaud, P., Absil, O., \& Surdej, J. 2005, ApJ, 633, 1191

Mawet, D., Serabyn, E., Liewer, K., et al. 2009, Opt. Express, 17, 1902

Mawet, D., Serabyn, E., Wallace, J. K., \& Pueyo, L. 2011, Opt. Lett., 36, 1506

Mazoyer, J., Baudoz, P., Galicher, R., Mas, M., \& Rousset, G. 2013a, A\&A, 557, A9

Mazoyer, J., Baudoz, P., Galicher, R., \& Rousset, G. 2013b, SPIE Conf. Ser., 8864

Mazoyer, J., Baudoz, P., Galicher, R., \& Rousset, G. 2014, A\&A, 564, L1

Murakami, N., Uemura, R., Baba, N., et al. 2008, PASP, 120, 1112

Patru, F., Baudoz, P., Galicher, R., et al. 2018, in Adaptive Optics Systems VI, SPIE Conf. Ser., 10703, 107032L

Potier, A., Baudoz, P., Galicher, R., Patru, F., \& Thijs, S. 2018, Proc. SPIE, 10698, 106986G

Racine, R., Walker, G. A. H., Nadeau, D., Doyon, R., \& Marois, C. 1999, PASP, 111,587

Raymond, S. N., Kokubo, E., Morbidelli, A., Morishima, R., \& Walsh, K. 2014, J., 595

Rouan, D. 2016, EAS Pub. Ser., 78, 73

Rouan, D., Riaud, P., Boccaletti, A., Clénet, Y., \& Labeyrie, A. 2000, PASP, 112, 1479

Santos, N. C. 2008, New Astron. Rev., 52, 154

Santos, N. C., Adibekyan, V., Figueira, P., et al. 2017, A\&A, 603, A30

Santos, N. C., \& Faria, J. P. 2018, Asteroseismology and Exoplanets: Listening to the Stars and Searching for New Worlds, 49, 165

Serabyn, E., Prada, C. M., Chen, P., \& Mawet, D. 2019, J. Opt. Soc. Am. B Opt. Phys., 36, D13

Singh, G., Galicher, R., Baudoz, P., et al. 2019, A\&A, 631, A106

Singh, G., Guyon, O., Baudoz, P., et al. 2014, Proc. SPIE, 9148

Snellen, I. A. G., Brandl, B. R., de Kok, R. J., et al. 2014, Nature, 509, 63

Snellen, I. A. G., de Kok, R. J., de Mooij, E. J. W., \& Albrecht, S. 2010, Nature, 465,1049

von Essen, C., Mallonn, M., Welbanks, L., et al. 2019, A\&A, 622, A71 